\title{
L-DUNFORD-PETTIS AND ALMOST L-DUNFORD-PETTIS SETS IN DUAL BANACH LATTICES
}

\author{
HALIMEH ARDAKANI ${ }^{1 *}$ AND MANIJEH SALIMI ${ }^{2}$ \\ ${ }^{1}$ Department of Mathematics, Payame Noor University, Iran \\ ${ }^{2}$ Department of Mathematics, Farhangian University, Iran \\ *Corresponding author: halimeh_ardakani@yahoo.com
}

\begin{abstract}
Following the concept of L-limited sets in dual Banach spaces introduced by Salimi and Moshtaghioun, we introduce the concepts of L-Dunford-Pettis and almost L-Dunford-Pettis sets in dual Banach lattices and then by a class of operators on Banach lattices, so called disjoint Dunford-Pettis completely continuous operators, we characterize Banach lattices in which almost L-Dunford-Pettis subsets of their dual, coincide with L-Dunford-Pettis sets.
\end{abstract}

\section{INTRODUCTION}

A subset $A$ of a Banach space $X$ is called limited (resp. Dunford-Pettis (DP)), if every weak* null (resp. weak null) sequence $\left(x_{n}^{*}\right)$ in $X^{*}$ converges uniformly on $A$, that is

$$
\lim _{n \rightarrow \infty} \sup _{a \in A}\left|\left\langle a, x_{n}^{*}\right\rangle\right|=0 .
$$

Also if $A \subseteq X^{*}$ and every weak null sequence $\left(x_{n}\right)$ in $X$ converges uniformly on $A$, we say that $A$ is an L-set. Every relatively compact subset of $E$ is DP. If every DP subset of a Banach space $X$ is relatively compact, then $X$ has the relatively compact DP property $\left(a b b . D_{r c} \mathrm{P}\right)$. For example, dual Banach spaces with the weak Radon-Nikodym property (see [11], in short WRNP) and Schur spaces (i.e., weak and norm

Received 2017-10-15; accepted 2017-12-16; published 2018-03-07.

2010 Mathematics Subject Classification. Primary 46A40; Secondary 46B40, 46B42.

Key words and phrases. Dunford-Pettis set; relatively compact Dunford-Pettis property; Dunford-Pettis completely continuous operator.

(C)2018 Authors retain the copyrights of their papers, and all open access articles are distributed under the terms of the Creative Commons Attribution License. 
convergence of sequences in $X$ coincide) have the $\mathrm{DP}_{r c} \mathrm{P}[6]$. Also we recall that a Banach space $X$ has the $\mathrm{DP}_{r c} \mathrm{P}$ if and only if every DP and weakly null sequence $\left(x_{n}\right)$ in $X$ is norm null.

Recently, the authors in [14], introduced the class of Dunford-Pettis completely continuous (abb. DPcc) operators on Banach spaces. In fact, a bounded linear operator $T: X \rightarrow Y$ between two Banach spaces is $D P c c$ if it carries DP and weakly null sequences in $X$ to norm null ones in $Y$. The class of all $D P c c$ operators from $X$ to $Y$ is denoted by $\operatorname{DPcc}(X, Y)$.

In this article, by the definition of L-limited sets in [12] in dual Banach spaces, we introduce the concepts of L-DP and almost L-DP sets in Banach lattices and then we obtain Banach lattices in which two classes of sets coincide. Finally by introducing the concept of disjoint DP completely continuous (abb. $D P^{d} c c$ ) operators between Banach lattices and positive $\mathrm{DP}_{r c} \mathrm{P}$, we obtain some characterizations of them and then the relation between the positive $\mathrm{DP}_{r c} \mathrm{P}$ of $E$ and $D P^{d} c c$ operators on $E$ is treated. The class of all $D P^{d} c c$ operators from $E$ to $Y$ is denoted by $D P^{d} c c(E, Y)$.

Here, we remember some definitions and terminologies from Banach lattice theory.

It is evident that if $E$ is a Banach lattice, then its dual $E^{*}$, endowed with the dual norm and pointwise order, is also a Banach lattice. The norm $\|$.$\| of a Banach lattice E$ is order continuous if for each generalized net $\left(x_{\alpha}\right)$ such that $x_{\alpha} \downarrow 0$ in $E,\left(x_{\alpha}\right)$ converges to 0 for the norm $\|$.$\| , where the notation x_{\alpha} \downarrow 0$ means that the net $\left(x_{\alpha}\right)$ is decreasing, its infimum exists and $\inf \left(x_{\alpha}\right)=0$. A Banach lattice $E$ is said to be $\sigma$-Dedekind complete if every countable subset of $E$ that is bounded above has a supremum. A subset $A$ of $E$ is called solid if $|x| \leq|y|$ for some $y \in A$ implies that $x \in A$ and the solid hull of $A$ is the smallest solid set containing $A$ and is exactly the set $\operatorname{Sol}(A)=\{y \in E:|y| \leq|x|$, for some $x \in A\}$.

Throughout this article, $X$ and $Y$ denote the arbitrary Banach spaces and $X^{*}$ refers to the dual of the Banach space $X$. We use $L_{w^{*}}\left(X^{*}, Y\right)$ for Banach spaces of all bounded weak*-weak continuous operators from $X^{*}$ to $Y$. Also $E$ and $F$ denote arbitrary Banach lattices and $E^{+}=\{x \in E: x \geq 0\}$ refers to the positive cone of the Banach lattice $E . B_{E}$ is the closed unit ball of $E$.

If $x$ is an element of a Banach lattice $E$, then absolute value of $x$ is denoted by $|x|$. If $a, b$ belong to $E$ and $a \leq b$, the interval $[a, b]$ is the set of all $x \in E$ such that $a \leq x \leq b$. A subset of a Banach lattice is called order bounded if it is contained in an order interval. A linear mapping $T$ from $E$ into $F$ is called order bounded if it carries order bounded subsets of $E$ into order bounded sets. We recall from [10] that, an element $x$ belonging to a Riesz space $E$ is discrete, if $x>0$ and $|y| \leq x$ implies $y=t x$ for some real number $t$. If every order interval $[0, y]$ in $E$ contains a discrete element, then $E$ is said to be a discrete Riesz space. The lattice operations in the Banach lattice $E$ are weakly sequentially continuous if for every weakly null sequence $\left(x_{n}\right)$ in $E,\left|x_{n}\right| \rightarrow 0$ for $\sigma\left(E, E^{*}\right)$. We refer the reader for undefined terminologies, to the classical references $[1],[2],[10]$. 


\section{2. (L)-Dunford-Pettis Sets in BAnaCh LATtices}

Following the introducing of the concept L-limited sets in [12], we define L-DP sets and we give some properties of them in Banach spaces and specially in Banach lattices. A norm bounded subset $B$ of a dual Banach space $X^{*}$ is said to be an L-limited set if every weakly null and limited sequence $\left(x_{n}\right)$ of $X$ converges uniformly to zero on the set $B$, that is $\sup _{f \in B}\left|f\left(x_{n}\right)\right| \rightarrow 0$.

Definition 2.1. A norm bounded subset $B$ of a dual Banach space $X^{*}$ is said to be an L-DP set if every weakly null and DP sequence $\left(x_{n}\right)$ of $X$ converges uniformly to zero on the set $B$, that is $\sup _{f \in B}\left|f\left(x_{n}\right)\right| \rightarrow 0$.

It is clear that every L-set set in $X^{*}$ is $\mathrm{L}-\mathrm{DP}$ and every subset of an $\mathrm{L}-\mathrm{DP}$ set is the same. Also, it is evident that every L-DP set is weak* bounded and so is bounded. Similar to [12, Theorem 2.2], we obtain:

(a) absolutely closed convex hull of an L-DP set is an L-DP set,

(b) relatively weakly compact subsets of dual Banach spaces are L-DP set,

(c) every weak* null sequence in dual Banach space is an L-DP set.

Note that the converse of assertion (b) in general, is false. In fact, the following theorem 2.1, shows that the closed unit ball of $\ell_{\infty}$ is an L-DP set.

Theorem 2.1. A Banach space $X$ has the $D P_{r c} P$ iff every bounded subset of $X^{*}$ is an $L-D P$ set.

Proof. Since the Banach space $X$ has the $\mathrm{DP}_{r c} \mathrm{P}$ iff every DP and weakly null sequence $\left(x_{n}\right)$ in $X$ is norm null [14], the proof is clear.

The following Theorem 2.2, gives a necessary and sufficient condition for Banach spaces that L-sets and L-DP sets in its dual coincide. We recall that an operator $T: X \rightarrow Y$ between two Banach spaces is completely continuous, if $T$ carries weakly null sequences in $X$ to norm null ones, and the class of completely continuous operators is denoted by $C c(X, Y)$.

Theorem 2.2. A Banach space $X$ has the DP property iff each L-DP set in $X^{*}$ is an $L$-set.

Proof. Suppose $X$ has the DP property. Since every weakly null sequence in $X$ is DP so every L-DP set in $X^{*}$ is an L-set.

Conversely, it is enough to show that for each Banach space $Y, C c(X, Y)=D P c c(X, Y)[14$, Theorem 1.5]. If $T: X \rightarrow Y$ is $D P c c$, it is clear that $T^{*}\left(B_{Y^{*}}\right)$ is an $\mathrm{L}-\mathrm{DP}$ set. So by hypothesis, it is an $\mathrm{L}-$ set and we know that the operator $T: X \rightarrow Y$ is completely continuous iff $T^{*}\left(B_{Y^{*}}\right)$ is an L-set.

Corollary 2.1. A Banach space with the DP ${ }_{r c} P$ has the DP property if and only if it has the Schur property.

Proof. It is clear that the Banach space $X$ has the Schur property if and only if every bounded subset of $X^{*}$ is $\mathrm{L}$-set. Now, if $X$ has the DP property and $\mathrm{DP}_{r c} \mathrm{P}$, then by Theorem 2.1 , unit ball $X^{*}$ is $\mathrm{L}-\mathrm{DP}$ and so it is an L-set. The converse of the assertion is also clear. 
Theorem 2.3. Let $A$ be an $L-D P$ subset of a dual Banach lattice $E^{*}$ and $E$ has the weakly sequentially continuous lattice operations. Then $|A|=\{|a|: a \in A\}$ is an $L-D P$ set.

Proof. We show that every weakly null and DP sequence $\left(x_{n}\right)$ in $E$ converges uniformly on $|A|$, that is, $\lim _{n \rightarrow \infty} \sup _{x^{*} \in A}\left|\left\langle x_{n},\left|x^{*}\right|\right\rangle\right|=0$.

From [10, Lemma 1.4.4], $\left\langle\left|x_{n}\right|,\left|x^{*}\right|\right\rangle=\max \left\{\left\langle z_{n}, x^{*}\right\rangle:\left|z_{n}\right| \leq\left|x_{n}\right|\right\}$ for all $n$. So, there exists $z_{n} \in E$, such that $\left|z_{n}\right| \leq\left|x_{n}\right|$ and $\left\langle\left|x_{n}\right|,\left|x^{*}\right|\right\rangle=\left\langle z_{n}, x^{*}\right\rangle$. Since $E$ has the weakly sequentially continuous lattice operations, the sequences $\left(\left|x_{n}\right|\right)$ and so $\left(z_{n}\right)$ are weakly null. Since the set $A$ is $\mathrm{L}-\mathrm{DP}, \sup _{x^{*} \in A}\left|\left\langle z_{n}, x^{*}\right\rangle\right| \rightarrow 0$. From $\sup _{x^{*} \in A}\left|\left\langle x_{n},\left|x^{*}\right|\right\rangle\right| \leq \sup _{x^{*} \in A}\left\langle\left|x_{n}\right|,\left|x^{*}\right|\right\rangle$, we have $\sup _{x^{*} \in A}\left|\left\langle x_{n},\left|x^{*}\right|\right\rangle\right| \rightarrow 0$ and then the set $|A|$ is L-DP.

Definition 2.2. A Banach space $X$ has the $L-D P$ property, if every $L-D P$ subset of $X^{*}$ is relatively weakly compact.

Theorem 2.4. For a Banach space $X$, the following are equivalent:

(a) $X$ has the $L-D P$ property,

(b) For each Banach space $Y, D P c c(E, Y)=W(E, Y)$,

(c) $\operatorname{DPcc}\left(X, \ell_{\infty}\right)=W\left(X, \ell_{\infty}\right)$.

Proof. $(a) \Rightarrow(b)$. Suppose that $X$ has the L-DP property and $T: X \rightarrow Y$ is DPcc. Thus $T^{*}\left(B_{Y^{*}}\right)$ is an L-DP set. So by hypothesis, it is relatively weakly compact and $T$ is a weakly compact operator.

$(b) \Rightarrow(c)$. It is obvious.

$(c) \Rightarrow(a)$. If $X$ does not have the $\mathrm{L}-\mathrm{DP}$ property, there exists an L-DP subset $A$ of $X^{*}$ that is not relatively weakly compact. So there is a sequence $\left(x_{n}\right) \subset A$ with no weakly convergent subsequence. Now we show that the operator $T: X \rightarrow \ell_{\infty}$ by

$$
T x=\left(\left\langle x, x_{n}^{*}\right\rangle\right), x \in X
$$

is $D P c c$, but it is not weakly compact. As $\left(x_{n}^{*}\right) \subset A$ is an L-DP set, for every weakly null and DP sequence $\left(x_{m}\right)$ in $X$ we have

$$
\left\|T x_{m}\right\|=\sup _{n}\left|\left\langle x_{m}, x_{n}^{*}\right\rangle\right| \rightarrow 0
$$

thus $T$ is a $D P c c$ operator. It is easy to see that

$$
T^{*}\left(e_{n}^{*}\right)=x_{n}^{*}, n \in N
$$

Thus $T^{*}$ is not a weakly compact operator and neithe is $T$. This finishes the proof.

The classical Banach lattices $\ell_{p}$, where $1 \leq p<\infty$ and Schur spaces are discrete $K B$-space and so they have the $\mathrm{DP}_{r c} \mathrm{P}$ [3]. The following corollary shows that the classical Banach lattices $\ell_{p}$, where $1<p<\infty$ have the L-DP property. 
Corollary 2.2. A Banach space with the $D P_{r c} P$ has the $L-D P$ property if and only if it is reflexive.

Proof. If a Banach space $X$ has the $\mathrm{DP}_{r c} \mathrm{P}$, then by [14], the identity operator on $X$ is $D P c c$ and so is weakly compact, thanks to the L-DP property of $X$. Hence $X$ is reflexive.

Recall that a Banach space $X$ is said to have the reciprocal DP property (abb. RDP) if every completely continuous operator on $X$ is weakly compact [7].

Theorem 2.5. If a Banach space $X$ has the $L-D P$ property, then it has the RDP.

Proof. For arbitrary Banach space $Y$, let $T: X \rightarrow Y$ be a completely continuous operator. Thus it is DPcc and so by Theorem 2.4, $T$ is weakly compact. Hence $X$ has the RDP property.

In the following, we show that the L-DP property is carried by every complemented subspace.

Theorem 2.6. If a Banach space $X$ has the $L-D P$ property, then every complemented subspace of $X$ has the $L-D P$ property.

Proof. Consider a complemented subspace $Y$ of $X$ and a projection map $P: X \rightarrow Y$. Suppose $P: Y \rightarrow \ell_{\infty}$ is a $D P c c$ operator, so $T P: X \rightarrow \ell_{\infty}$ is also DPcc. Since $X$ has the L-DP property, by Theorem 2.4, TP is weakly compact. Hence $T$ is weakly compact.

The following evident proposition gives a characterization of the L-DP property property by L-DP setes.

Proposition 2.1. Let $X$ be a Banach space. Then the following are equivalent:

(a) $X$ has the $L-D P$ property,

(b) Every $L-D P$ sequence in $X^{*}$ is relatively weakly compact.

Theorem 2.7. Let $E$ be a Banach lattice with the $L-D P$ property. Then for each $f \in\left(E^{*}\right)^{+},[-f, f]$ is an $L-D P$ set.

Proof. It is evident that every L-set in $E^{*}$ is an L-DP set. If $E$ is a Banach lattice $E$ with the L-DP property, then every L-set in $E^{*}$ is relatively weakly compact and so by [?, Theorem 3.1], $E^{*}$ has an order continuous norm. Hence by [2, Theorem 4.9], for each $f \in\left(E^{*}\right)^{+},[-f, f]$ is relatively weakly compact and so it is an L-DP set.

In the rest of this section by using some techniques to those in [4], we investigate additional properties of L-DP sets.

Proposition 2.2. Let $X$ be a Banach space and $B$ be a bounded subset of $X^{*}$. Then the following are equivalent: 
(a) $B$ is an $L-D P$ set,

(b) For each sequence $\left(f_{n}\right)$ in $B, f_{n}\left(x_{n}\right) \rightarrow 0$, for every weakly null and DP sequence $\left(x_{n}\right)$ of $X$.

Proof. $(a) \Rightarrow(b)$. This is from the inequality $\left|f_{n}\left(x_{n}\right)\right| \leq \sup _{f \in B}\left|f\left(x_{n}\right)\right|$ for each sequence $\left(f_{n}\right)$ in $B$ and for every weakly null and DP sequence $\left(x_{n}\right)$ of $X$.

$(b) \Rightarrow(a)$. Assume that $B$ is not an (L) DP set in $X^{*}$. Then there exsits an $\epsilon>0$ and a weakly null and DP sequence $\left(x_{n}\right)$ in $X$ such that $\sup _{f \in B}\left|f\left(x_{n}\right)\right|>\epsilon$ for all $n$. This implies the existence of a sequence $f_{n}$ in $B$ such that $\left|f_{n}\left(x_{n}\right)\right|>\epsilon$, for all $n$.

As in the previous proposition 2.2, we can easily conclude that, for a norm bounded sequence $\left(f_{n}\right)$ of $X^{*}$, the subset $\left\{f_{n}: n \in N\right\}$ is an L-DP set iff $f_{n}\left(x_{n}\right) \rightarrow 0$, for every weakly null and DP sequence $\left(x_{n}\right)$ of $X$.

Proposition 2.3. Let $T$ be an operator from a Banach space $X$ into a Banach lattice $E$ and $f \in\left(E^{*}\right)^{+}$. Then the following are equivalent:

(a) $T^{*}[-f, f]$ is an $L-D P$ set,

(b) For every weakly null and DP sequence $\left(x_{n}\right)$ of $X, f\left(\left|T\left(x_{n}\right)\right|\right) \rightarrow 0$.

Proof. It follows immediately from the equality $f\left(\left|T\left(x_{n}\right)\right|\right)=\sup _{g \in T^{*}[-f, f]}\left|g\left(x_{n}\right)\right|$.

By taking $T=I d_{E}$ in Proposition 2.3, for each $f \in\left(E^{*}\right)^{+},[-f, f]$ is an L-DP set iff for every weakly null and DP sequence $\left(x_{n}\right)$ of $E,\left(\left|x_{n}\right|\right)$ is weakly null.

The next main result, gives an equivalent condition to $T^{*}(B)$ be an L-DP set, where $B$ is a norm bounded solid subset of $E^{*}$ and $T$ is an operator from a Banach space $X$ into a Banach lattice $E$. Recall that a sequence $\left(x_{n}\right)$ in a Banach lattice $E$ is (pairwise) disjoint, if for each $i \neq j,\left|x_{i}\right| \wedge\left|x_{j}\right|=0$.

Theorem 2.8. Let $T$ be an operator from a Banach space $X$ into a Banach lattice $E$ and $B$ be a norm bounded solid subset of $E^{*}$. Then the following are equivalent:

(a) $T^{*}(B)$ is an $L-D P$ set in $X^{*}$,

(b) $T^{*}[-f, f]$ and $\left\{T^{*} f_{n}: n \in N\right\}$ are $L-D P$ sets, for each $f \in B^{+}$and for each norm bounded disjoint sequence $\left(f_{n}\right) \in B^{+}$.

Proof. The proof is similar to [4, Theorem 2.7].

By taking $T=I d_{E}$ in Theorem 2.8, we obtain a norm bounded solid subset $B$ of $E^{*}$ is an L-DP set iff $[-f, f]$ and $\left\{f_{n}: n \in N\right\}$ are L-DP sets, for each $f \in B^{+}$and for each disjoint sequence $\left(f_{n}\right) \in B^{+}$.

The next result characterizes $D P c c$ operators by L-DP sets.

Theorem 2.9. For an operator $T$ from a Banach space $X$ into a Banach lattice $E$, the following are equivalent: 
(a) $T$ is DPcc,

(b) $T^{*}\left(B_{E^{*}}\right)$ is an $L-D P$ set, where $B_{E^{*}}$ is the closed unit ball of $E^{*}$,

(c) $T^{*}[-f, f]$ and $\left\{T^{*} f_{n}: n \in N\right\}$ are $L-D P$ sets, for each $f \in\left(B_{E^{*}}\right)^{+}$and for each norm bounded disjoint sequence $\left(f_{n}\right) \in\left(B_{E^{*}}\right)^{+}$,

(d) $\left|T\left(x_{n}\right)\right| \rightarrow 0$ for $\sigma\left(E, E^{*}\right)$ and $f_{n}\left(T x_{n}\right) \rightarrow 0$, for every weakly null and DP sequence $\left(x_{n}\right)$ in $X$ and for each disjoint sequence $\left(f_{n}\right)$ in $\left(B_{E^{*}}\right)^{+}$.

Proof. $(a) \Leftrightarrow(b)$. By the equality $\sup _{f \in T^{*}\left(B_{E}^{*}\right)}\left|f\left(x_{n}\right)\right|=\left\|T x_{n}\right\|_{E}, T^{*}\left(B_{E^{*}}\right)$ is an L-DP set in $X^{*}$, if and only if, $T$ is a $D P c c$ operator.

By Theorem 2.8, the statements (b) and (c) are equivalent and the equivalence $(c) \Leftrightarrow(d)$ is a direct consequence of Proposition 2.3.

\section{Almost L-DP sets in Banach lattices}

In this section we introduce a new class of sets and operators.

Definition 3.1. Let $E$ be a Banach lattice and $X$ be a Banach space. Then

(a) A norm bounded subset $B$ of a dual Banach lattice $E^{*}$ is said to be an almost L-DP set if every disjoint weakly null and DP sequence $\left(x_{n}\right)$ of $E$ converges uniformly to zero on the set $B$, that is $\sup _{f \in B}\left|f\left(x_{n}\right)\right| \rightarrow 0$.

(b) An operator $T$ from a Banach lattice $E$ into a Banach space $X$ is a disjoint DP completely continuous (abb. $\left.D P^{d} c c\right)$ operator if the sequence $\left(\left\|T x_{n}\right\|\right)$ converges to zero for every disjoint weakly null and $D P$ sequence in $E$.

Note that every L-DP set of a dual Banach lattice, is an almost L-DP set, but the converse is false, in general. In fact for many Banach lattices $E$ with the positive $\mathrm{DP}_{r c} \mathrm{P}$ and without the $\mathrm{DP}{ }_{r c} \mathrm{P}$, the closed unit ball of the dual Banach lattice $E^{*}$ is an almost L-DP set, but it is not L-DP set. As an example, the closed unit ball $B_{\ell_{\infty}}$ of $\ell_{\infty}$ is an almost $\mathrm{L}-\mathrm{DP}$ set in $\ell_{\infty}$, but the closed unit ball $B_{\left(\ell_{\infty}\right)}$ is not an almost L-DP set in $\left(\ell_{\infty}\right)^{*}$. In the following, we give a useful chracterization of almost L-DP sets, that is proved by the method of Proposition 2.2.

As we mentioned at the end of the previous section, we use some techniques to those in [4].

Proposition 3.1. Let $E$ be a Banach lattice and $B$ be a norm bounded set in $E^{*}$. Then the following are equivalent:

(a) $B$ is an almost $L-D P$ set,

(b) For each sequence $\left(f_{n}\right)$ in $B, f_{n}\left(x_{n}\right) \rightarrow 0$, for every disjoint weakly null and DP sequence $\left(x_{n}\right)$ of E. 
In particular, we obtain:

Proposition 3.2. Let $E$ be a Banach lattice and $\left(f_{n}\right)$ be a norm bounded sequence in $E^{*}$. Then the following are equivalent:

(a) The subset $\left\{f_{n}: n \in N\right\}$ is an almost $L-D P$ set,

(b) $f_{n}\left(x_{n}\right) \rightarrow 0$, for every disjoint weakly null and DP sequence $\left(x_{n}\right)$ of $E$.

Similar to [4], $[-f, f]$ is an almost L-DP set in $E^{*}$, for each $f \in\left(E^{*}\right)^{+}$. Also for an order bounded operator from a Banach lattice $E$ into a Banach lattice $F, T^{*}([-f, f])$ is an almost L-DP set, for each $f \in\left(F^{*}\right)^{+}$.

Theorem 3.1. Let $T$ be an order bounded operator from a Banach lattice $E$ into a Banach lattice $F$ and $B$ be a norm bounded solid subset of $F^{*}$. Then the following are equivalent:

(a) $T^{*}(B)$ is an almost $L-D P$ set in $E^{*}$,

(b) $\left\{T^{*} f_{n}: n \in N\right\}$ is an almost $L-D P$ set, for each $f \in B^{+}$and for each disjoint sequence $\left(f_{n}\right)$ in $B^{+}$.

(c) $f_{n}\left(T x_{n}\right) \rightarrow 0$, for every disjoint weakly null and DP sequence $\left(x_{n}\right)$ of $E^{+}$and for each disjoint sequence $\left(f_{n}\right)$ in $B^{+}$.

Proof. The proof is the same as the proof of Theorem 2.9.

By taking $T=I d_{E}$ in Theorem 3.1, we obtain a norm bounded solid subset $B$ of $E^{*}$ is an almost L-DP set iff $\left\{f_{n}: n \in N\right\}$ is an almost $\mathrm{L}-\mathrm{DP}$ set for each disjoint sequence $\left(f_{n}\right)$ in $B^{+}$.

The next result characterizes the class of $D P^{d} c c$ operators by almost L- DP sets.

Theorem 3.2. For an order bounded operator $T$ from a Banach lattice $E$ into another Banach lattice $F$, the following are equivalent:

(a) $T$ is $D P^{d} c c$,

(b) $T^{*}\left(B_{F^{*}}\right)$ is an almost $L-D P$ set, where $B_{F^{*}}$ is the closed unit ball of $F^{*}$,

(c) $\left\{T^{*}\left(f_{n}\right): n \in N\right\}$ is an almost $L-D P$ set for each disjoint sequence $\left(f_{n}\right)$ in $\left(B_{F^{*}}\right)^{+}$,

(d) $f_{n}\left(T\left(x_{n}\right)\right) \rightarrow 0$, for every disjoint weakly null and DP sequence $\left(x_{n}\right)$ of $E^{+}$and for each disjoint sequence $\left(f_{n}\right)$ in $\left(B_{F^{*}}\right)^{+}$.

Proof. $(a) \Leftrightarrow(b)$. By the equality, $\sup _{f \in T^{*}\left(B_{F^{*}}\right)}\left|f\left(x_{n}\right)\right|=\left\|T x_{n}\right\|_{F}$, for every sequence $\left(x_{n}\right)$ in $E$, it follows easily that, $T^{*}\left(B_{F^{*}}\right)$ is an almost L-limited set in $E^{*}$ if and only if $T$ is $D P^{d} c c$.

By Theorem 3.1, the statements (b) and (c) are equivalent and the equivalence $(c) \Leftrightarrow(d)$ is a direct consequence of Proposition 3.2. 
Now the concept of positive $\mathrm{DP}_{r c} \mathrm{P}$ in Banach lattices is introduced and Banach lattices with the positive $\mathrm{DP}_{r c} \mathrm{P}$ is characterized. Next we give some properties of $D P^{d} c c$ operators from an arbitrary Banach lattice $E$ to another $F$, related to the positive $\mathrm{DP}_{r c} \mathrm{P}$ of the Banach lattice $E$.

Definition 3.2. A Banach lattice $E$ has the positive $D P_{r c} P$ if each weakly null and DP sequence with the positive terms in E is norm null.

It is clear that the $\mathrm{DP}_{r c} \mathrm{P}$ implies the positive $\mathrm{DP}_{r c} \mathrm{P}$, but the converse is false, in general. For example, $L^{1}[0,1]$ has the positive $\mathrm{DP}_{r c} \mathrm{P}$ without the $\mathrm{DP}_{r c} \mathrm{P}$.

Theorem 3.3. For a Banach lattice E, the following are equivalent:

(a) E has the positive $D P_{r c} P$,

(b) Every weakly null and disjoint DP sequence in E converges to zero in norm.

Proof. $(a) \Rightarrow(b)$. Let $\left(x_{n}\right)$ be a weakly null and disjoint DP sequence in $E$. From [15, Proposition 1.3$]$, the sequence $\left(\left|x_{n}\right|\right)$ is weakly null and by $\left[8\right.$, Lemma 3.7], it is DP in $E$. From (a), the sequence $\left(\left|x_{n}\right|\right)$ and so $\left(x_{n}\right)$ converges to zero in norm.

$(b) \Rightarrow(a)$. Suppose that $\inf _{n}\left\|x_{n}\right\|=c>0$ for some weakly null and DP sequence $\left(x_{n}\right) \subset E^{+}$. Putting $y_{n}=c^{-1} x_{n}$ and using [9, Corollary 5] we find a subsequence $\left(y_{n_{k}}\right)$, a constant $d>0$, and a disjoint sequence $\left(z_{k}\right)$ of $E^{+}$such that $0<z_{k} \leq y_{n_{k}}$ and $\left\|z_{k}\right\| \geq d$. It is clear that disjoint DP sequence $\left(z_{k}\right)$ tends weakly to zero, but $\left\|z_{k}\right\| \geq d$. This fact contradicts the assumption.

Theorem 3.4. A Banach lattice $E$ has the positive $D P_{r c} P$ iff every bounded set in $E^{*}$ is an almost L-DP set.

Proof. From Theorem 3.3, a Banach lattice $E$ has the positive $\mathrm{DP}_{r c} \mathrm{P}$ iff every disjoint weakly null and DP sequence in $E$ is norm null.

Theorem 3.5. Let $E$ be a Banach lattice. Then the following are equivalent:

(a) E has the positive $D P_{r c} P$,

(b) For each Banach space $Y, D P^{d} c c(E, Y)=L(E, Y)$,

(c) $D P^{d} c c\left(E, \ell_{\infty}\right)=L\left(E, \ell_{\infty}\right)$.

Proof. $(a) \Rightarrow(b)$. If $E$ has the positive $\mathrm{DP}_{r c} \mathrm{P}$ and $\left(x_{n}\right)$ is a weakly null and disjoint $\mathrm{DP}$ sequence in $E$, then by Theorem 3.3, $\left(x_{n}\right)$ is norm null and for each bounded operator $T$ on $E$, $\left\|T x_{n}\right\| \rightarrow 0$; that is, $D P^{d} c c(E, F)=L(E, F)$.

$(b) \Rightarrow(c)$. It is obvious.

$(c) \Rightarrow(a)$. If $E$ does not have the positive $\mathrm{DP}_{r c} \mathrm{P}$, then by Theorem 3.3 , there exists a weakly null and 
disjoint DP sequence $\left(x_{n}\right)$ in $E$ such that $\left\|x_{n}\right\|=1$, for all $n$. Choose a normalized sequence $\left(x_{n}^{*}\right)$ in $E^{*}$ such that $\left|\left\langle x_{n}, x_{n}^{*}\right\rangle\right|=1$, for all $n$, and define the operator $T: E \rightarrow \ell_{\infty}$ by

$$
T x=\left(\left\langle x, x_{n}^{*}\right\rangle\right), x \in E .
$$

But $T$ is not $D P^{d} c c$, since the sequence $\left(x_{n}\right)$ is weakly null and disjoint DP and $\left\|T x_{n}\right\| \geq 1$, for all $n$.

In the following Theorem 3.6, we show that the positive $\mathrm{DP}_{r c} \mathrm{P}$ and the $\mathrm{DP}_{r c} \mathrm{P}$, coincide in the class of discrete Banach lattices. Let us start with the following lemma.

Lemma 3.1. $c_{0}$ dose not have the positive $D P_{r c} P$.

Proof. It is enough to remember that $c_{0}$ dose not have the positive Schur property and use the fact that every weakly null sequence in $c_{0}$ is DP. By [13], a Banach lattice has the positive Schur property, whenever $0 \leq x_{n} \rightarrow 0$ weakly implies $\left\|x_{n}\right\| \rightarrow 0$

Now we are able to formulate the following equivalence condition.

Theorem 3.6. Let $E$ be a discrete Banach lattice. Then $E$ has the positive $D P_{r c} P$, if and only if, it has the $D P_{r c} P$.

Proof. Since the positive $\mathrm{DP}_{r c} \mathrm{P}$ is inherited by closed Riesz subspaces and $c_{0}$ does not have the positive $\mathrm{DP}_{r c} \mathrm{P}$, then $E$ does not contain any order copy of $c_{0}$. According to [10, Corollary 2.4.12], $E$ is $K B$ space, and so it possesses the $\mathrm{DP}_{r c} \mathrm{P}$ by [?].

Corollary 3.1. The dual Banach lattice $C(K)^{*}$ has the positive $D P_{r c} P$, where $K$ is a compact Hausdorff space.

Proof. For each positive and weakly null sequence $\left(f_{n}\right)$ in $C(K)^{*},\left\|f_{n}\right\|=f_{n}\left(1_{K}\right) \rightarrow 0$, where $1_{K}$ denotes the constant function 1 on $K$. That is $C(K)^{*}$ has the positive $\mathrm{DP}_{r c} \mathrm{P}$. On the other hands from [2], the Banach lattice $C(K)^{*}$ is discrete and by Theorem 3.6, it has the $\mathrm{DP}_{r c} \mathrm{P}$.

Theorem 3.7. Let $T: E \rightarrow X$ from a Banach lattice $E$ be an operator. Then the following are equivalent:

(a) $T$ is $D P^{d} c c$,

(b) the sequence $\left(\left\|T x_{n}\right\|\right)$ converges to zero for every weakly null and DP sequence in $E^{+}$,

(c) the sequence $\left(\left\|T x_{n}\right\|\right)$ converges to zero for every disjoint weakly null and DP sequence in $E^{+}$.

Proof. The proof is similar to [5, Theorem 2.2].

Let $\mathcal{M} \subset L(X, Y)$ be a Banach lattice. If $\mathcal{M}$ has the positive $\mathrm{DP}_{r c} \mathrm{P}$, then by Theorem 3.5 all of the evaluation operators $\phi_{x}$ and $\psi_{y^{*}}$ are $D P^{d} c c$ operators, where $\phi_{x}(T)=T x$ and $\psi_{y^{*}}(T)=T^{*} y^{*}$ for $x \in X$, 
$y^{*} \in Y^{*}$ and $T \in \mathcal{M}$. Now, we show that the $D P^{a} c c$ ness of evaluation operators is a sufficient condition for the positive $\mathrm{DP}_{r c} \mathrm{P}$ of their domain.

Theorem 3.8. Let $Y$ has the Schur property and $\mathcal{M} \subset L(X, Y)$ be a Banach lattice. If for every $y^{*} \in Y^{*}$, the evaluation operator $\psi_{y^{*}}$ on $\mathcal{M}$ is $D P^{d} c c$, then $\mathcal{M}$ has the positive $D P_{r c} P$.

Proof. If $\mathcal{M}$ does not have the positive $\mathrm{DP}_{r c} \mathrm{P}$, by Theorem 3.3, there exists a weakly null and disjoint $\mathrm{DP}$ sequence $\left(T_{n}\right)$ in $\mathcal{M}$ and some $\epsilon>0$ such that $\left\|T_{n}\right\|>\epsilon$, for all $n$. So there exists a sequence $\left(x_{n}\right)$ in $B_{X}$ such that $\left\|T_{n}\left(x_{n}\right)\right\|>\epsilon$, for all $n$. On the other hand, the evaluation operator $\psi_{y^{*}}$ on $\mathcal{M}$ is $D P^{d} c c$ for all $y^{*} \in Y^{*}$ and so $\left\|T_{n}^{*}\left(y^{*}\right)\right\|=\left\|\psi_{y^{*}}\left(T_{n}\right)\right\| \rightarrow 0$. Hence $\left|\left\langle T_{n} x_{n}, y^{*}\right\rangle\right| \leq\left\|T_{n}^{*}\left(y^{*}\right)\right\| \rightarrow 0$. So the sequence $\left(T_{n} x_{n}\right)$ is weakly null and it is norm null by the Schur property, a fact that is impossible.

Theorem 3.9. Let $X$ has the Schur property and $\mathcal{M} \subset L_{w^{*}}\left(X^{*}, Y\right)$ be a Banach lattice. If for every $x^{*} \in X^{*}$, the evaluation operator $\phi_{x^{*}}$ on $\mathcal{M}$ is $D P^{d} c c$, then $\mathcal{M}$ has the positive $D P_{r c} P$.

Proof. If $\mathcal{M}$ does not have the positive $\mathrm{DP}_{r c} \mathrm{P}$, by Theorem 3.3, there exists a weakly null and disjoint DP sequence $\left(T_{n}\right)$ in $\mathcal{M}$ and some $\epsilon>0$ such that $\left\|T_{n}\right\|>\epsilon$, for all $n$. On the other hand, the evaluation operator $\phi_{x^{*}}$ on $\mathcal{M}$ is $D P^{d} c c$ for all $x^{*} \in X^{*}$ and so $\left\|T_{n}\left(x^{*}\right)\right\|=\left\|\phi_{x^{*}}\left(T_{n}\right)\right\| \rightarrow 0$. Since $\left\|T_{n}^{*}\right\|>\epsilon$, there exists a sequence $\left(y_{n}^{*}\right)$ in $B_{Y^{*}}$ such that $\left\|T_{n}^{*} y_{n}^{*}\right\|>\epsilon$, for all $n$. But the Schur property of $X$ shows that the weakly null sequence $\left(T_{n}^{*} y_{n}^{*}\right)$ is norm null, which is a contradiction.

Two final theorems of this section, are a relationship between order weakly compact and $M$-weakly compact operators with a $D P^{d} c c$ operator.

Recall that a continuous operator $T: E \rightarrow X$ from a Banach lattice $E$ to a Banach space $X$ is order weakly compact if and only if $\left\|T x_{n}\right\| \rightarrow 0$ for every disjoint order bounded sequence $\left(x_{n}\right)$ in $E[2$, Theorem 5.57$]$.

Theorem 3.10. Evere $D P^{d}$ cc operator on a Banach lattice $E$ is order weakly compact.

Proof. Let $\left(x_{n}\right)$ be an order bounded disjoint sequence of $E$. It follows from [2] and [?] that $\left(x_{n}\right)$ is a weakly null and DP sequence. Since $T$ is $D P^{d} c c$ then, $\left\|T x_{n}\right\| \rightarrow 0$. Hence $T$ is order weakly compact.

An operator $T: E \rightarrow X$ from a Banach lattice to a Banach space is said to be $M$-weakly compact if $\left\|T x_{n}\right\| \rightarrow 0$ holds for every norm bounded disjoint sequence $\left(x_{n}\right)$ in $E$ [10]. In [14], the authors proved that each $D P c c$ operator from a Banach lattice $E$ to a Banach space $X$ is $M$-weakly compact when $E^{*}$ has an order continuous norm and $E$ has the $D P^{*}$ property (that is, every relatively weakly compact set in $E$ is limited). In fact, we have a similar conclusion about $D P^{d} c c$ operators.

Theorem 3.11. Let $E$ be a Banach lattice and $X$ be a Banach space. If $E^{*}$ has an order continuous norm and $E$ has the DP property, then each $D P^{d}$ cc operator $T: E \rightarrow X$ is $M$-weakly compact. 
Proof. Let $T: M \rightarrow X$ be a $D P^{d} c c$ operator and let $\left(x_{n}\right)$ be a bounded disjoint sequence in $E$. It follows from [10, Corollary 2.9] that $\left(x_{n}\right)$ is weakly null and so it is DP by the DP property of E. By our hypothesis on $T$, we have $\left\|T x_{n}\right\| \rightarrow 0$ and then $T$ is $M$-weakly compact.

\section{Almost L-DP sets Which are L-DP sets}

As we noted in the beginning of section 3, every L-DP set in the dual Banach lattice $E^{*}$, is an almost L-DP set, but the converse is false in general. In this section we characterize Banach lattices in which the class of almost L-DP sets and that of L-DP sets coincide in their dual.

Theorem 4.1. For a Banach lattice E, the following are equivalent:

(a) Each almost $L-D P$ set in $E^{*}$ is an $L-D P$ set,

(b) For each Banach space $Y, D P^{d} c c(E, Y)=D P c c(E, Y)$,

(c) $D P^{d} c c\left(E, \ell_{\infty}\right)=\operatorname{DPcc}\left(E, \ell_{\infty}\right)$.

Proof. $(a) \Rightarrow(b)$. Let $T: E \rightarrow Y$ be an operator. By the equality

$$
\sup _{f \in T^{*}\left(B_{Y^{*}}\right)}\left|f\left(x_{n}\right)\right|=\left\|T x_{n}\right\|_{Y}
$$

for every sequence $\left(x_{n}\right)$ in $E$, it follows easily that, $T^{*}\left(B_{Y^{*}}\right)$ is an almost L-DP (respectively, L-DP) set in $E^{*}$, if and only if, $T$ is a $D P^{d} c c$ (respectively, $D P c c$ ) operator. Now, let $T$ be a $D P^{d} c c$ operator. Then $T^{*}\left(B_{Y^{*}}\right)$ is an almost L-DP set in $E^{*}$ and from the hypothesis (a), it is an L-DP set in $E^{*}$. Hence $T$ is a DPcc operator.

$(b) \Rightarrow(c)$. It is clear.

$(c) \Rightarrow(a)$. Let $B$ be an almost $\mathrm{L}-\mathrm{DP}$ set in $E^{*}$. To prove that $B$ is an $\mathrm{L}-\mathrm{DP}$ set, it sufficies to show that $f_{n}\left(x_{n}\right) \rightarrow 0$ for each sequence $\left(f_{n}\right)$ in $B$ and for every weakly null and DP sequence $\left(x_{n}\right)$ in $E($ see Proposition 2.2). Consider the operator $S: E \rightarrow \ell_{\infty}$ defined by $S(x)=\left(f_{n}(x)\right)_{n=1}^{\infty}$, for each $x \in E$. As $B$ is almost L-DP, $S$ is a $D P^{d} c c$ operator. In fact, for every weakly null and disjoint DP sequence $\left(z_{i}\right)$ in $E$, we have

$$
\left\|S z_{i}\right\|_{\infty}=\left\|f_{n}\left(z_{i}\right)_{n=0}^{\infty}\right\|_{\infty} \leq \sup _{f \in B}\left|f\left(z_{i}\right)\right| \rightarrow 0
$$

as $i \rightarrow \infty$. It follows that $S$ is a $D P^{d} c c$ operator and so from our hypothesis, $S$ is $D P c c$. So $\left\|S x_{n}\right\|_{\infty} \rightarrow 0$ and the desired conclusion follows from the inequality $\left|f_{n}\left(x_{n}\right)\right| \leq\left\|S x_{n}\right\|_{\infty}$ for each $n$.

We recall that, an operator $T$ from a Banach space $X$ into a Banach lattice $E$ is said to be semicompact if for each $\epsilon>0$ there exists some $u \in E^{+}$satisfying $T\left(B_{X}\right) \subset[-u, u]+\epsilon B_{E}$. According to [4, Theorem 4.3], each operator $T: E \rightarrow X$ is $D P^{d} c c$, whenever its adjoint $T^{*}: X^{*} \rightarrow E^{*}$ is semicompact. 
At the end of this section, it should be noted that the adjoint of a $D P^{d} c c$ operator is not necessary $D P^{d} c c$ and vice versa. For example, the identity operator on the Banach lattice $\ell_{1}$ is $D P^{d} c c$ (because $\ell_{1}$ has the $\left.\mathrm{DP}_{r c} \mathrm{P},[14]\right)$ but its adjoint, $I d_{\ell_{\infty}}: \ell_{\infty} \rightarrow \ell_{\infty}$, is not $D P^{d} c c$. In fact, if $e_{n}=(0,0, \ldots, 1,0, \ldots)$ with n'th entry equals to 1 and all others zero, then $\left(e_{n}\right)$ is an order bounded disjoint sequence of $\ell_{\infty}$. Hence $\left(e_{n}\right)$ is weakly null and by [?], it is DP, but $\left\|I d_{\ell_{\infty}}\left(e_{n}\right)\right\|=\left\|e_{n}\right\|_{\infty}=1$ for all $n$. Also the identity operator on $\ell_{\infty}$ is not $D P^{d} c c$ but its adjoint is $D P^{d} c c$, because $\left(\ell_{\infty}\right)^{*}$ has $\mathrm{DP}_{r c} \mathrm{P}$. Also by Theorem $3.2, B_{\left(\ell_{\infty}\right)^{*}}$ is not an almost $\mathrm{L}-\mathrm{DP}$ set in $\left(\ell_{\infty}\right)^{*}$, as noted that in the begining of section 3 .

\section{REFERENCES}

[1] C. D. Aliprantis and O. Burkishaw, Locally Solid Riesz Spaces, Academic Press, New York, London, 1978.

[2] C. D. Aliprantis and O. Burkishaw, Positive Operators, Academic Press, New York, London, 1978.

[3] B. Aqzzouz and K. Bouras, Dunford-Pettis sets in Banach lattices, Acta Math. Univ. Comenianae, 81 (2012), $185-196$.

[4] B. Aqzzouz and K. Bouras, L-sets and almost L- sets in Banach lattices, Quaest. Math., 36 (2013), $107-118$.

[5] B. Aqzzouz and A. Elbour, Some characterizations of almost Dunford-Pettis operators and applications, J. Positivity 15 (2011), 369-380.

[6] G. Emmanuele, Banach spaces in which Dunford-Pettis sets are relatively compact, Arch. Math., 58 (1992), $477-485$.

[7] G. Emmanuele, The reciprocal Dunford-Pettis property and projective tensor products, Math. Proc. Cambridge Philos. Soc., 109 (1992), 161-166.

[8] K.E. Fahri, N. Machrafi and M. Moussa, Banach Lattices with the Positive Dunford-Pettis Relatively Compact Property, Extracta Math., 80 (2015), 161-179.

[9] G. Groenewegen and P. Meyer-Nieberg, An elementary and unified approach to disjoint sequence theorems, Indag. Math., 48 (1986), 313-317.

[10] P. Meyer- Nieberg, Banach Lattices, Universitext, Springer- Verlag, Berlin, 1991.

[11] K. Musial, The weak Radon-Nikodym property in Banach spaces, Studia Math., 64 (1979), 151-173.

[12] M. Salimi and S. M. Moshtaghioun, A new class of Banach spaces and its relation with some geometric properties of Bancah spaces, Abstr. Appl. Anal., ID 212957, 2012.

[13] J. A. Sanchez, Positive Schur property in Banach lattices, Extraccta Math., 7 (1992), 161-163.

[14] Y. Wen, Ji. Chen, Characterizations of Banach spaces with relatively compact Dunford-Pettis sets, Adv. Math., to appear.

[15] W. Wnuk, On the dual positive Schur property in Banach lattices, J. Positivity, 17 (2013), 759-773. 